\title{
Pain aversion and anxiety-like behavior occur at different times during the course of chronic inflammatory pain in rats
}

This article was published in the following Dove Press journal:

Journal of Pain Research

6 November 2017

Number of times this article has been viewed

\section{Yuanyuan $\mathrm{Wu}^{\prime}$ \\ Xinmiao $\mathrm{Yao}^{2}$ \\ Yongliang Jiang' \\ Xiaofen $\mathrm{He}^{\prime}$ \\ Xiaomei Shao' \\ Junying $\mathrm{Du}^{\prime}$ \\ Zui Shen' \\ Qiaoying $\mathrm{He}^{\mathrm{l}}$ \\ Jianqiao Fang'}

'Department of Neurobiology and Acupuncture Research, The Third Clinical Medical College, Zhejiang

Chinese Medical University, Hangzhou,

${ }^{2}$ The Third Affiliated Hospital of

Zhejiang Chinese Medical University,

Zhejiang, China
Correspondence: Jianqiao Fang Department of Neurobiology and Acupuncture Research, The Third Clinical Medical College, Zhejiang Chinese Medical University, 548 Binwen Road, Binjiang District, Hangzhou, Zhejiang 310053, China

Tel +8657186673000

Email fangjianqiao7532@163.com

\begin{abstract}
Pain is considered a multidimensional conscious experience that includes a sensory component and a negative affective-motivational component. The negative affective-motivational component of pain is different from the sensory component and amplifies the pain experience. Nowadays, a significant number of preclinical research groups have focused their attention on the affective symptoms of pain. In the present study, we investigated the pain aversion and anxietylike behavior of the complete Freund's adjuvant (CFA)-induced chronic pain model. CFA rats experienced spontaneous pain during pain-paired conditioning (pain aversion) and spontaneous pain produces an affective response (anxiety-like behavior). Moreover, pain aversion was gradually attenuated, while the anxiety-like behavior increased in 4 weeks. Therefore, although the negative effect (including pain aversion and anxiety) is always associated with hyperalgesia, the manifestations of negative effect may follow different time courses, which may influence the progress of primary disease. The findings illustrate that targeted therapy should focus on a specific aspect in different stages of pain. Our study emphasizes the necessity of using multiple tests to study pain comorbidities.
\end{abstract}

Keywords: pain, pain aversion, pain affection, anxiety-like behavior, chronic inflammatory pain, rat

\section{Introduction}

Pain is considered a multidimensional conscious experience that includes a sensory component (perception of the severity and location of the pain) and a negative affectivemotivational component (pain aversion and emotional affect). ${ }^{1}$

Clinical patients suffering from pain usually exhibit varying degrees of affective symptoms such as anxiety, depression, and aversion to pain-associated environments. ${ }^{2,3}$ The negative affective-motivational component of pain is different from the sensory component ${ }^{4,5}$ and amplifies the pain experience. Recent surveys report that arthritis is one of the most common chronic pain conditions in North America, affecting $\sim 16 \%$ of the adult population in the United States and Canada. ${ }^{6}$ Furthermore, anxiety symptoms related to arthritis have also been shown to increase pain.

In the last two decades, a myriad of behavioral paradigms have been introduced in the field of experimental pain research, particularly in chronic pain. ${ }^{7-9}$ Recently, a significant number of preclinical research groups have focused their attention on the affective symptoms of pain. ${ }^{10-12}$ The conditioned-place aversion (CPA) paradigm is a highly sensitive method for measuring the negative effect of an aversive memory associated with a pain-paired context. ${ }^{13-16}$ Recent studies have shown that the affective component of chronic inflammatory pain can be assessed with a CPA test (pain aver- 
sion) and other emotional behavior tests, such as the elevated zero maze (EZM) and open field (OF) tests. ${ }^{17,18}$ However, the onset, development, and maintenance of the affective component remain unclear.

In the present study, we investigated the complete Freund's adjuvant (CFA)-induced chronic pain model using a CPA test and assessed anxiety-like behavior with the EZM and OF tests. We hypothesized that the time of onset and development tendency would differ between pain aversion and pain-induced anxiety.

\section{Materials and methods}

\section{Animals}

Male Sprague-Dawley rats (250 $\pm 20 \mathrm{~g})$ were purchased from the Shanghai Laboratory Animal Center (SLAC, Shanghai, China) and housed in $40 \times 50 \times 25 \mathrm{~cm}$ cages with ad libitum access to food and water at room temperature $\left(25 \pm 1^{\circ} \mathrm{C}\right)$. The animals were housed in groups of 5-6 rats with a 12:12 lightdark cycle (dark cycle 8:00 PM to 8:00 AM). Our experiments were approved by Laboratory Animal Management and Use Committee of Zhejiang Chinese Medical University. All the animal experiments were performed in accordance with the regulations of the State Science and Technology Commission for the Care and Use of Laboratory Animals (State Science and Technology Commission No. 2, 1988).

\section{Experimental design}

Rats were randomly divided into two groups: 1) a salineinjected group (saline, $n=8$ ) and 2) a CFA-injected group (CFA, n=32), with subgroups including the day 7 (D7), day 14 (D14), day 21 (D21), and day 28 (D28) groups. On day 1, the baseline paw withdrawal threshold was assessed before the saline/CFA injection and repeated on days 7, 14, 21, and 28. On day 2, CPA tests were conducted on each rat. The CPA test, EZM test, and OF test were administered to the D7, D14, D21, and D28 groups. The timeline is shown in Figure 1A.

\section{Arthritis pain model and sham controls}

Inflammatory pain was induced with an intraplantar injection of $100 \mu \mathrm{L}$ of CFA in the plantar surface of the left hind paw of the rats. Each milliliter of the injection contained $1 \mathrm{mg}$ of heat-killed and dried Mycobacterium tuberculosis (ATCC $25177), 0.85 \mathrm{~mL}$ of paraffin oil and $0.15 \mathrm{~mL}$ of mannide monooleate (F5881; Sigma-Aldrich, St Louis, MO, USA). Control animals were injected with the same volume of $0.9 \%$ saline.

\section{CPA paradigm}

The CFA-induced CPA (C-CPA) test was performed as previously reported using a place conditioning apparatus made of Plexiglas. ${ }^{19}$ The apparatus consisted of two equal square compartments, $30 \mathrm{~cm}$ on each side, positioned on the floor and separated by guillotine doors. No neutral third chamber was present in the center. One compartment was covered on three sides with 30 white dots, $1.8 \mathrm{~cm}$ in diameter, at $3.5 \mathrm{~cm}$ intervals; the floor of the compartment was covered with cinnamon oil. The other compartment was covered with 23 white equilateral triangles with a side length of $2.5 \mathrm{~cm}$; the floor of the compartment was covered with $5 \%$ acetic acid. The area of the compartment with 30 white dots was the same as the area of the compartment with 23 white triangles. The guillotine doors, covered with colored
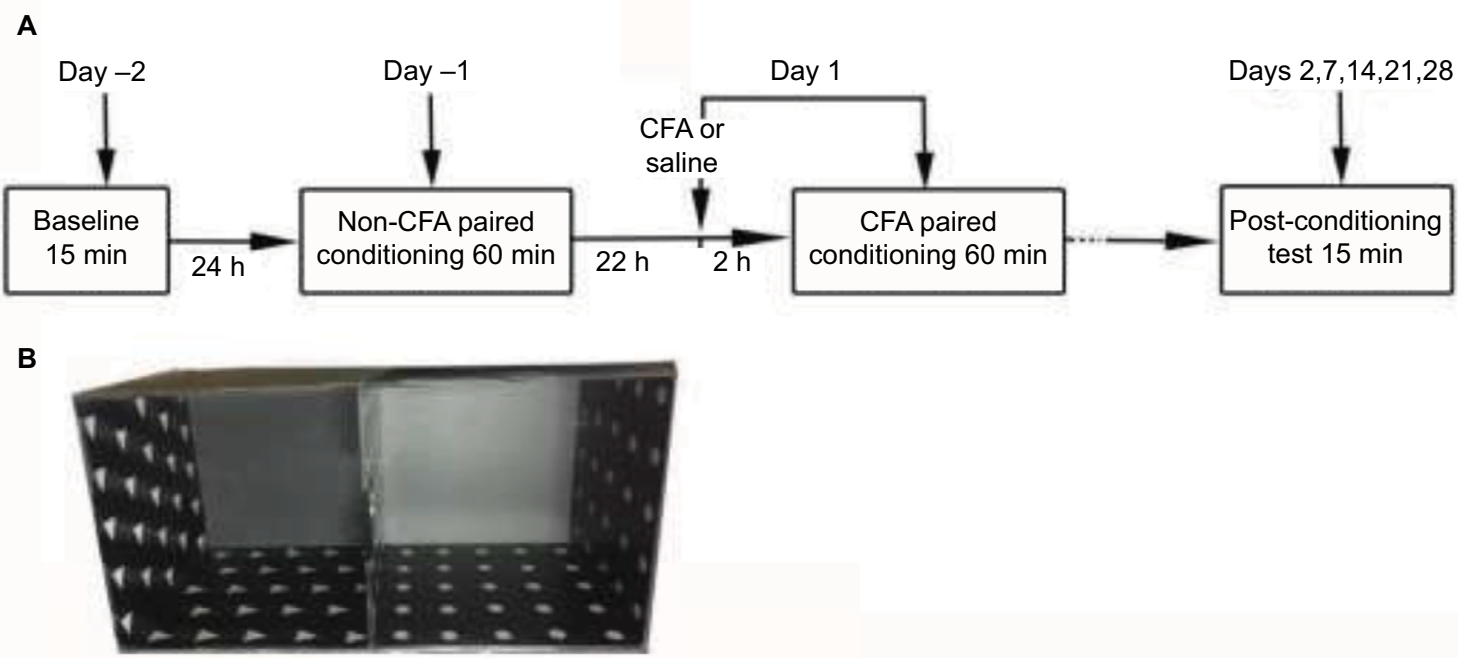

Figure I Schematic diagram of the protocol for C-CPA testing. (A) Procedure for the C-CPA test and (B) the place conditioning apparatus made of Plexiglas. Abbreviations: CFA, complete Freund's adjuvant; C-CPA, CFA-induced conditioned-place aversion. 
spots corresponding to their respective walls, were inserted during conditioning sessions and removed during pre- and post-conditioning tests. The colored dots and triangle spots served as the visual cues; the different substances on the floor served as the tactile cues (Figure 1B). The testing room was illuminated with a $15-\mathrm{W}$ bulb positioned $\sim 1 \mathrm{~m}$ from the apparatus. The apparatus was cleaned with $75 \%$ ethanol after each test.

\section{Pre-conditioning phase}

In the pre-conditioning phase (day -2$)$, the baseline time that the rats spent during a 15 -min pre-conditioning period in each of the two distinctive compartments was recorded. The animal was considered to be in a chamber when the midpoint of the back was inside the chamber. The next day (day -1$)$, each rat was free to explore in one compartment for 1 hour.

\section{Conditioning phase}

In the conditioning phase (day -1 ), each rat was free to explore one compartment for 1 hour. For the C-CPA group, CFA was subcutaneously injected in the plantar surface of left hind paw (day 1). Two hours after the injection, the rat was free to explore another compartment for another hour. The pain-paired compartment was assigned randomly before the baseline measurement.

\section{Testing phase}

During the 15-min post-conditioning phase (day 2), the amount of time that the rats spent in each compartment was recorded again. The CPA score magnitude, used as an indicator of affective response, was determined by subtracting the time spent in the pain-paired compartment during the post-conditioning test (day 2) from the time spent in the same compartment during the pre-conditioning test (day-2). Less post-conditioning time spent in the compartment indicated a greater affective response. Additional tests were performed on days $7,14,21$, and 28 . The timeline is shown in Figure 1A.

\section{Assessment of static mechanical sensitivity}

The rats were habituated to the testing chambers two to three times before the baseline testing began. The testing chambers consisted of clear Plexiglas chambers on a raised wire mesh grid. On each testing day, the rats were first habituated to the testing chambers 30 minutes before the test. Mechanical allodynia, as a behavioral sign of neuropathic pain, was assessed by measuring 50\% paw withdrawal threshold (PWT) as described by Dixon. ${ }^{20}$
Mechanical threshold measurements for each hind paw were obtained. For this procedure, eight von Frey monofilaments $(1.4,2,4,6,8,10,15$, and $26 \mathrm{~g})$ were utilized in the following manner. Each trial began with a von Frey force of $8 \mathrm{~g}$ delivered to the left hind paw for $\sim 5$ seconds. If there was no withdrawal response, the next higher force was delivered. If there was a response, the next lower force was delivered. This procedure was performed until no response was made at the highest force (26 g) or until four stimuli were administered following the initial response. The 50\% PWT was calculated using the following formula: PWT= $10[\mathrm{Xf}+\mathrm{k} \delta]$, where $\mathrm{Xf}$ is the value of the final von Frey filament used (in $\log$ units), $\mathrm{k}$ is a value measured from the pattern of positive/negative responses, and $\delta=0.184$, which is the average interval (in log units) between the von Frey filaments. If an animal responded to the lowest von Frey filament, a value of $1.4 \mathrm{~g}$ was assigned. If an animal did not respond to the highest von Frey filament, the value was recorded as $15.0 \mathrm{~g}$. The tests were conducted on days 1 (baseline), 7, 14, 21, and 28.

Pain-induced emotional and affective changes were assessed in a separate set of animals on days 7, 14, 21, and 28 after the CFA injection.

\section{EZM test}

The maze consisted of a black metallic annular platform (100 cm in diameter, $25 \mathrm{~cm}$ in width, and $55 \mathrm{~cm}$ in height) that was equally divided into four quadrants. Two opposite quadrants (close arms) were enclosed by black metallic walls (30 cm high) on both the inner and outer edges of the platform, while the remaining two opposite quadrants (open arms) were open. ${ }^{21}$ All the animals were placed in the testing room 1 hour before the test. The whole apparatus was wiped with $75 \%$ ethanol before each trial. The animal was placed in the center of a closed arm for 20 seconds to adapt to the environment, and then the behavior was videotaped and quantified by the SMART 3.0 system for 5 minutes. The EZM test was administered to a separate set of animals on days 7 (D7 group), 14 (D14 group), 21 (D21 group), and 28 (D28 group) after the CFA injection.

\section{OF test}

Four square, black Plexiglas OF arenas $(100 \times 100 \times 50 \mathrm{~cm})$ were placed together to form the apparatus, such that each side was $200 \mathrm{~cm}$, and the area of the four arenas was included. The whole apparatus was wiped with $75 \%$ ethanol before each trial. ${ }^{22,23}$ All the animals were placed in the testing room 1 hour before the test to adapt to the environment. At 
the beginning of the trial, each animal was first placed in the center of the area, and the behavior was videotaped for 5 minutes, which was quantified by the SMART 3.0 system. The OF test was performed on a separate set of animals on days 7 (D7 group), 14 (D14 group), 21 (D21 group), and 28 (D28 group) after the CFA injection.

\section{Statistical analysis}

All data are expressed as the mean \pm standard error of the mean. Statistical comparisons among different groups were made by one-way repeated-measures analysis of variance (ANOVA). For the subsequent multiple comparisons, the least significant difference (LSD) and Dunnett's test were used when equal variances were assumed or not assumed, respectively, as determined by the homogeneity of variance test. Correlations between the CPA scores and anxiety-like behavior were determined using Pearson correlation coefficient analysis. Significance was determined at the level of $p<0.05$.

\section{Results \\ CFA injection into the left hind paw induced an affective response in the C-CPA test}

During the 15-minute pre-conditioning test, rats in the saline and CFA groups spent similar amounts of time in the two compartments, indicating no preference for either compartment. However, when a hind-paw CFA injection was paired with a specific compartment, the rats spent less time in the paired compartment during the post-conditioning test than during the pre-conditioning test, which demonstrated place aversion to the paired compartment (Figure 2A). In contrast, saline-injected rats spent similar amounts of time in both the compartments during the post-conditioning and pre-conditioning tests, which indicated no aversion to the saline-paired compartment (Figure 2B). The CPA score revealed that the apparatus chambers were neutral stimuli and demonstrated that the CFA injection induced pain affection compared to the saline injection (Figure 2C).

A
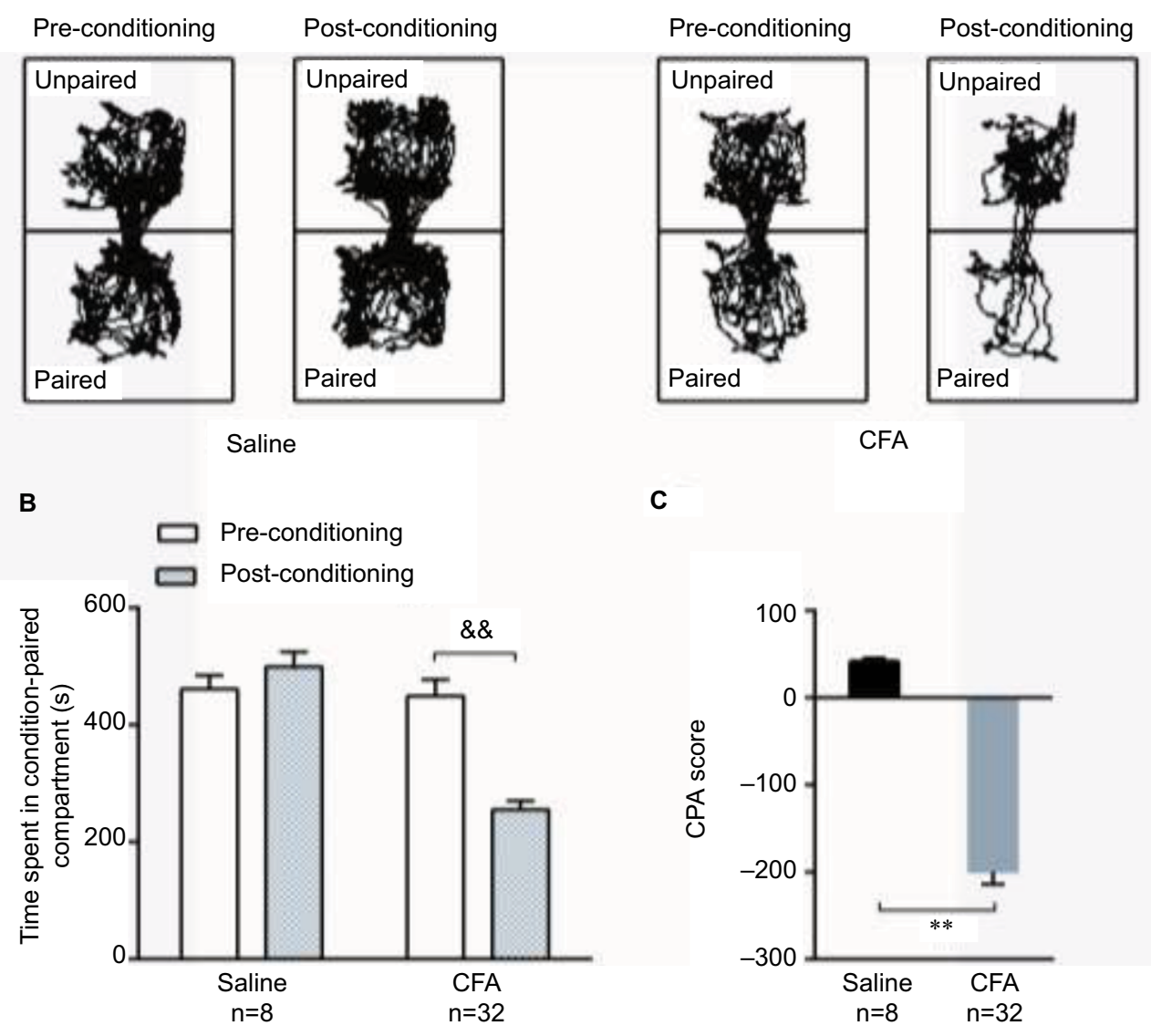

C

CFA

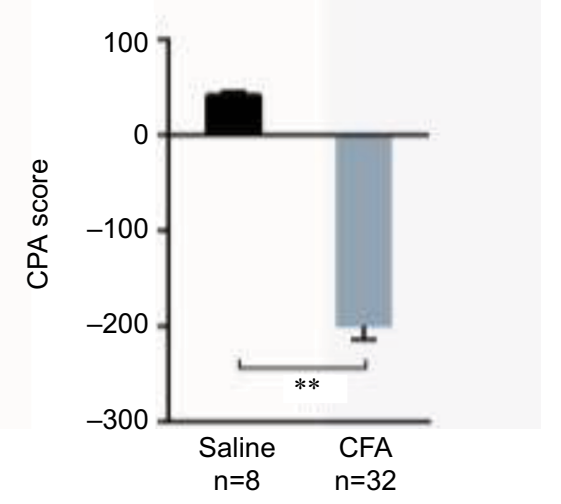

Figure 2 C-CPA tests. (A) Trajectory chart of rats in the pre-conditioning and post-conditioning tests with saline or CFA injections. (B) The time spent in the conditionpaired compartment before and after the conditioning phase. (C) The CPA scores of the saline group and CFA group on day 2 were determined by subtracting the time spent in the pain-paired compartment during the post-conditioning test. ${ }^{2 \times} p<0.01$, post-conditioning compared to pre-conditioning; ${ }^{* *} p<0.01$, compared to the saline group. Abbreviations: CFA, complete Freund's adjuvant; C-CPA, CFA-induced conditioned-place aversion. 


\section{Development of mechanical hypersensitivity following CFA injection}

Unilateral intraplantar injection of both the CFA solution and saline into the rat hind paw caused a decrease in the PWT after the application of the mechanical stimulus to the ipsilateral side of the rat. Mechanical hypersensitivity was evident due to the obvious paw swelling at day 7 (Figure 3A), and significant decreases in the PWT (Figure 3B) were observed in the CFA-injected rats compared to the saline-treated rats. In the saline group, the PWT exhibited a temporary decline and then recovered to the baseline level. Mechanical allodynia remained 28 days after the CFA injection and represented a significant $(80 \%)$ decrease in the mechano-nociceptive threshold of the affected limb compared to the saline-treated animals. Overall, the CFA induced persistent mechanical allodynia in the CFA-treated hind paw.

\section{Time course of CFA-induced pain aversion in the CPA test}

After the CPA tests (day 2), the CFA rats were divided into D7, D14, D21, and D28 groups, and another CPA test was conducted on days 7, 14, 21, and 28, respectively (Figure 4A). A significant decrease was observed in the CPA score on day 7 after the CFA injection and gradually increased on days 14,21 , and 28 but was still less than that of the salineinjected group (Figure 4B).

\section{CFA injection induced anxiety-like behavior on day 28 in the EZM test}

On days 7,14 , and 21 , the traveling distance and time in the open arms were decreased in the CFA-injected group, but no significant differences were observed compared to the saline-injected group (Figure 5C). On day 28, the CFAinjected rats traveled shorter distances in the open arms than the saline-injected rats (Figure 5B). There is no difference in the locomotor activity (Figure 5D). Trajectory charts of rats by EZM tests are shown in (Figure 5A). These data indicate that rats with long-term inflammatory pain exhibit anxietylike behavior at $\sim 4$ weeks after pain initiation but not during the early onset of pain.

\section{Time course of CFA-induced anxiety-like behavior in the OF test}

To verify the results in the EZM tests, we examined OF behavior (Figure 6A). The animals explored freely in each arena for 5 minutes, and the distance traveled and time spent in the center were analyzed. The CFA injection induced a decrease in the time spent in the central area and distance traveled. No significant differences were observed on days 7 and 14 after the CFA injection. However, on days 21 and 28 , the rats displayed a significant decrease in the time spent in the center and distance traveled, which serve as indices of anxiety in rodents (Figure 6B and C). There is no difference in the distance traveled (Figure 6D).

\section{Correlation between pain aversion and pain-induced anxiety behaviors}

To investigate the seemingly inconsistent results for pain aversion and pain-induced anxiety, Pearson correlations were calculated between the CPA score and anxiety-like behaviors in the EZM tests as well as between the CPA score and anxiety-like behaviors in the OF tests. Significant nega-
A

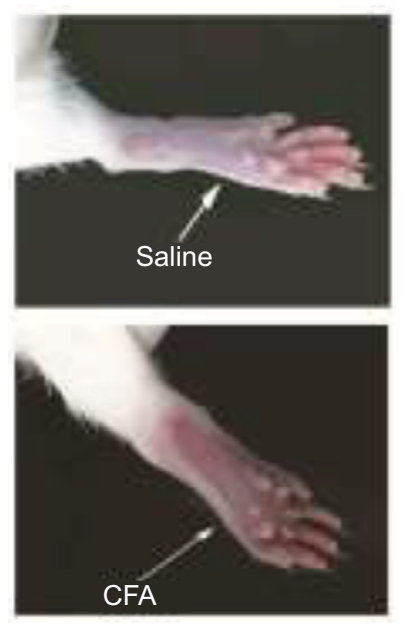

B



Figure 3 (A) Photographs of the hind paws reveal obvious swelling and the guarding posture of the CFA rats. (B) Development of nociceptive behavior in saline and CFA rats. All values are expressed as the mean \pm SEM: ${ }^{* *} p<0.01$, compared to saline controls, $n=8$ per experimental group. Abbreviations: CFA, complete Freund's adjuvant; SEM, standard error of the mean; PWT, paw withdrawal threshold. 
A


Figure 4 The variation of CFA-induced pain aversion in the C-CPA tests. (A) Trajectory chart of the rats in the D7, DI4, D21, and D28 saline groups in the postconditioning tests. (B) CPA score. ${ }^{* *} p<0.01$, compared to the saline group, ${ }^{*} p<0.05$, compared to the saline group.

Abbreviations: CFA, complete Freund's adjuvant; C-CPA, CFA-induced conditioned-place aversion.

tive correlations were identified between the CPA score and anxiety-like behaviors in the EZM tests $(r=0.7316, p<0.0001$, Figure 7A) and OF tests $(r=0.7263, p<0.0001$, Figure 7B).

\section{Discussion}

In the present study, plantar injection of CFA stimulated a rapid, local inflammatory response that caused both periph- eral and central sensitization; CFA-induced pain was intense and persisted for at least 4 weeks after the injection. Moreover, the CFA injection induced affective pain compared to the saline injection, as shown by the avoidance response to the pain-paired compartment over 4 weeks. The data indicate that CFA rats experienced spontaneous pain during pain-paired conditioning and that CFA injection-induced spontaneous pain produces an affective response.

Isolated chronic pain is a debilitating phenomenon that affects $\sim 20 \%$ of the population. ${ }^{24}$ Common comorbidities, such as cognitive, anxiety, and sleep disorders, are reported in most cases. ${ }^{25}$ Over the past decade, a number of research groups have explored the changes in emotional affect caused by chronic pain in rodents using pain models such as viralinduced neuropathy, drug-induced toxic neuropathy, nerve injury-induced neuropathy, or models mimicking arthritic pain. ${ }^{18,26-28}$ Sawada et al have reported that in measurements of anxiety-like behavior, the time spent in the open arms for partial sciatic nerve ligations-treated mice decreased only on day 28 , and no differences was found on days 7,14 , and $21 .{ }^{27}$ These studies have shown that the development of pain influences the onset of anxiety/depressive-like behaviors in rodents, which are similar with our study.

The C-CPA test is based on associative learning between a painful stimulus aversion and the environmental context in which the stimulus is administered; the inhibition of painrelated aversive behavior may be caused by a disruption of various forms of long-term memory. ${ }^{15,16}$ In our study, C-CPA was gradually attenuated to the baseline level over 4 weeks, but we still observed significant aversion to the pain-paired

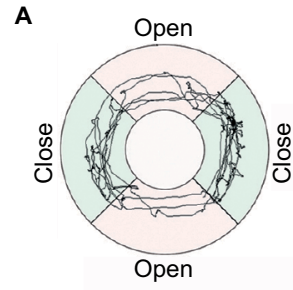

Saline

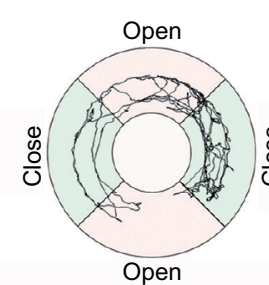

D7

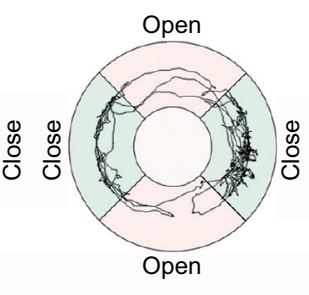

D14



D21

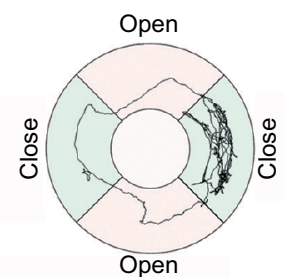

D28
B

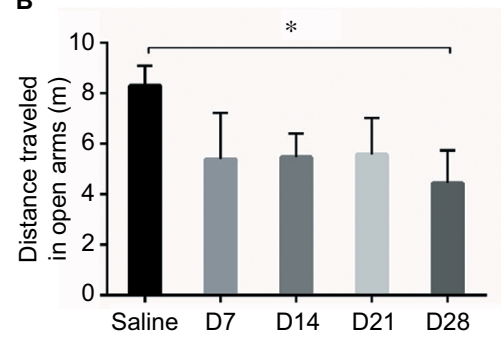

C

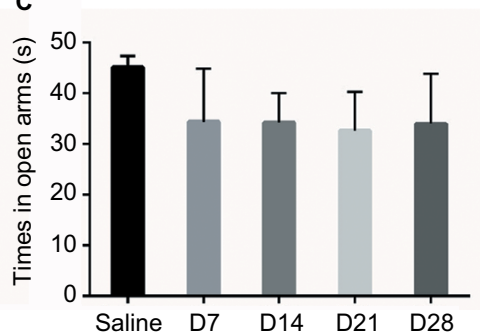

D

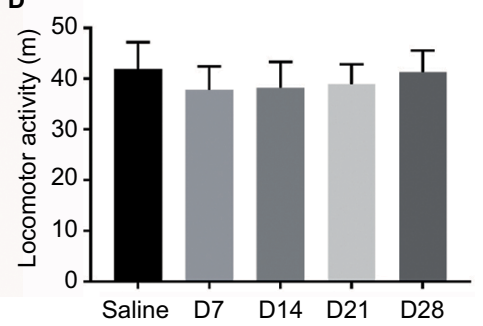

Figure 5 The EZM test. (A) Trajectory chart of the rats in the EZM. (B) The distance traveled in the open arms of the EZM. (C) The time spent in the open arms of the EZM. (D) Locomotor activity of the rats in the EZM. ${ }^{*} p<0.05$, compared to the saline group.

Abbreviation: EZM, elevated zero maze. 




Saline

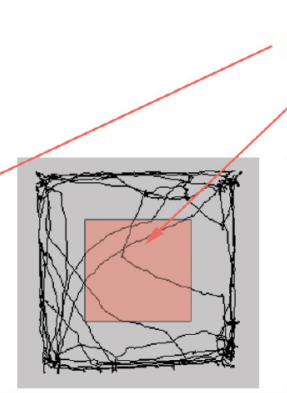

D7
Central area

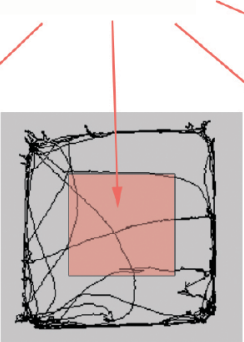

D14

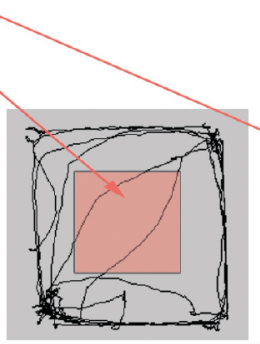

D21



D28
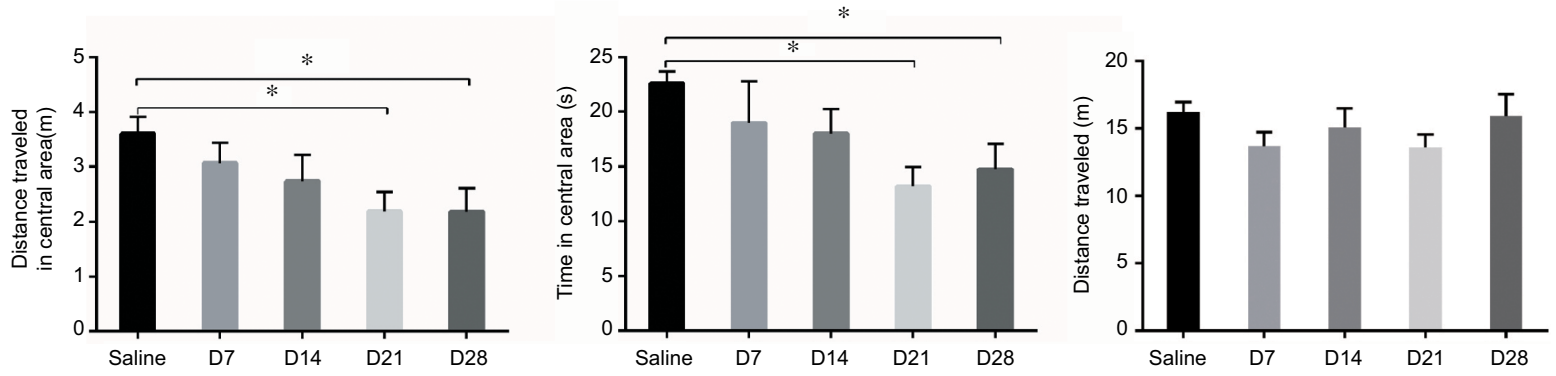

Figure 6 The OF test. (A) Trajectory chart of the rats in the OF test. (B) The distance traveled in the central area of the OF. (C) The time spent in central area of the OF. (D) Distance traveled in the OF. ${ }^{*}<<0.05$, compared to the saline group. Abbreviation: OF, open field.

A

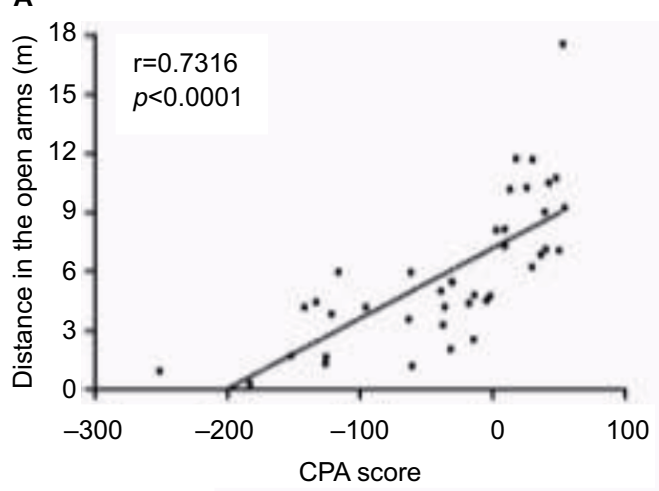

B

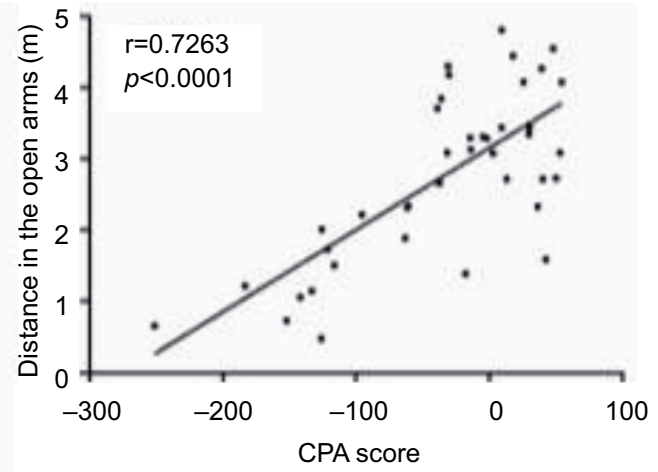

C

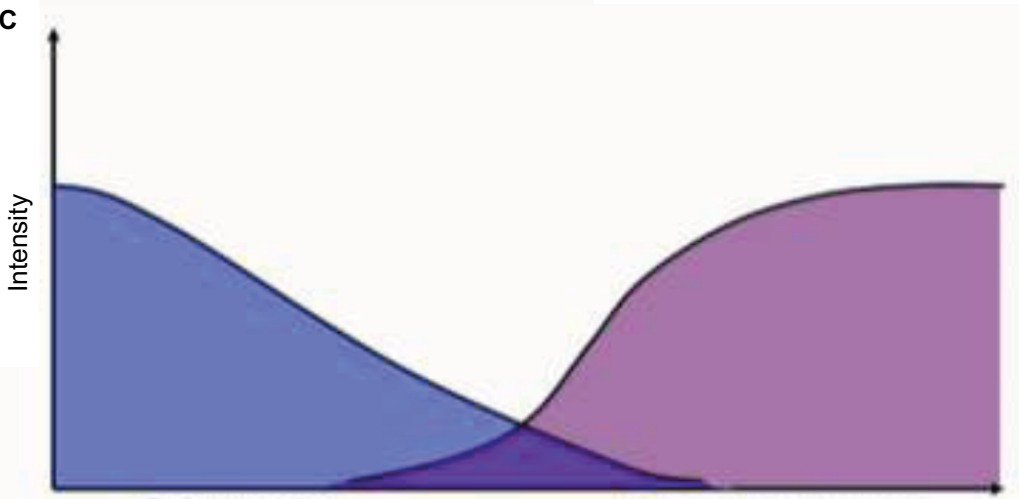

Pain aversion

Pain-induced anxiety

Figure 7 The relationship between pain aversion and pain-induced anxiety. (A) The distance traveled in the open arms of the EZM test was positively correlated with the CPA score. (B) The distance traveled in the central area of the OF test was positively correlated with the CPA score. (C) The overlap of pain aversion and pain-induced anxiety over time. At the early stage, CFA rats exhibit strong pain aversion in the C-CPA tests. This aversion slowly decreased over time, while the onset of pain-induced anxiety occurred and increased over time.

Abbreviations: CFA, complete Freund's adjuvant; C-CPA, CFA-induced conditioned-place aversion; EZM, elevated zero maze; OF, open field. 
compartment in the CFA-injected group compared to the saline group. These findings indicate that the association between the painful stimulus aversion and the environmental context was weakened over time. Human patients with chronic pain often exhibit higher levels of anxiety. In our study, whether the emotional effect was also attenuated remains unknown. Therefore, we used the EZM and OF tests to assess the anxiety-like behavior of the animals in our study.

Because anxiety cannot be directly assessed in animals, most tests (eg, EZM and OF) used in preclinical studies rely on evaluating exploratory behaviors that are believed to be associated with anxiety (anxiety-like behavior). ${ }^{29,30}$ Here, we addressed the behavioral impact of persistent pain in animals by monitoring the affective state behaviors using intraplantar CFA injections. Indeed, in rats suffering from unilateral inflammation, anxiety-related phenotypes were revealed by a decrease in the distance traveled and time spent in the center of the OF and in the open arms of the EZM at 3 and 4 weeks after the CFA injection. However, no anxiety-like behavior was detected at 1 or 2 weeks after the CFA injection. Hence, we demonstrated that chronic inflammatory pain leads to the development of anxiety-like behaviors at 3 or 4 weeks after pain induction.

In the present study, the CFA-induced inflammatory pain in these rats was accompanied by the attenuation of painrelated aversion in the C-CPA test and the development of anxiety-like behaviors in the EZM and OF tests. The C-CPA test induces affective responses through associative learning and memory formation, which reflects an emotional affect related to episodic memory. As episodic memory was abated over time, the aversion to pain was correspondingly attenuated. In addition, the anxiety-like behavior that reflected a pure emotional affect was increased, even as the episodic memory was attenuated (Figure 7C).

These disparities reinforce the hypothesis that although negative effect (including pain aversion and anxiety) is always associated with hyperalgesia, the manifestations of negative effect may follow different time courses, which emphasizes that targeted therapy should focus on specific aspect in different stages of pain. The findings illustrate the necessity of using multiple tests to study pain comorbidities.

\section{Acknowledgments}

This work was supported by the National Natural Science Foundation of China (81603691) and the Medicine and Health Science and Technology Plan projects of Zhejiang province (2017183595). This manuscript has been edited and proofread by Nature Publishing Group Language Editing.

\section{Disclosure}

The authors report no conflicts of interest in this work.

\section{References}

1. Moseley GL, Butler DS. Fifteen years of explaining pain: the past, present, and future. J Pain. 2015;16(9):807-813.

2. Gerrits MM, van Oppen P, van Marwijk HW, Penninx BW, van der Horst HE. Pain and the onset of depressive and anxiety disorders. Pain. 2014;155(1):53-59.

3. Wieser MJ, Gerdes AB, Reicherts P, Pauli P. Mutual influences of pain and emotional face processing. Front Psychol. 2014;5:1160.

4. Jennings EM, Okine BN, Roche M, Finn DP. Stress-induced hyperalgesia. Prog Neurobiol. 2014;121:1-18.

5. Linton SJ, Bergbom S. Understanding the link between depression and pain. Scand J Pain. 2011;2(2):47-54.

6. Giesecke T, Gracely RH, Williams DA, Geisser ME, Petzke FW, Clauw DJ. The relationship between depression, clinical pain, and experimental pain in a chronic pain cohort. Arthritis Rheum. 2005;52(5): $1577-1584$.

7. Rouwette T, Sondermann J, Avenali L, Gomez-Varela D, Schmidt M. Standardized profiling of the membrane-enriched proteome of mouse dorsal root ganglia (DRG) provides novel insights into chronic pain. Mol Cell Proteomics. 2016;15(6):2152-2168.

8. Sung HJ, Kim YS, Kim IS, et al. Proteomic analysis of differential protein expression in neuropathic pain and electroacupuncture treatment models. Proteomics. 2004;4(9):2805-2813.

9. Sui P, Watanabe H, Ossipov MH, Bakalkin G, Artemenko K, Berqquist J. Proteomics of neuropathic pain: proteins and signaling pathways affected in a rat model. J Proteome Res. 2014;13(9):3957-3965.

10. Burke NN, Finn DP, Roche M. Neuroinflammatory mechanisms linking pain and depression. Mod Trends Pharmacopsychiatry. 2015;30:36-50.

11. Wu YY, Jiang YL, He XF, et al. Effects of electroacupuncture with dominant frequency at SP 6 and ST 36 based on meridian theory on pain-depression dyad in rats. Evid Based Complement Alternat Med. 2015;2015:732845.

12. Shao XM, Sun J, Jiang YL, et al. Inhibition of the cAMP/PKA/CREB pathway contributes to the analgesic effects of electroacupuncture in the anterior cingulate cortex in a rat pain memory model. Neural Plast. 2016;2015(2015):5320641.

13. Minami M. Neuronal mechanisms for pain-induced aversion behavioral studies using a conditioned place aversion test. Int Rev Neurobiol. 2009;85:135-144.

14. Minami M. Neuronal mechanisms underlying pain-induced negative emotions. Brain Nerve. 2012;64(11):1241-1247. Japanese.

15. Zhang L, Wang G, Ma J, et al. Brain-derived neurotrophic factor (BDNF) in the rostral anterior cingulate cortex (rACC) contributes to neuropathic spontaneous pain-related aversion via NR2B receptors. Brain Res Bull. 2016;127:56-65.

16. Lu B, Jiang J, Sun J, et al. Inhibition of mammalian target of rapamycin activation in the rostral anterior cingulate cortex attenuates pain-related aversion in rats. Behav Brain Res. 2016;310:51-58.

17. Zhang RX, Zhang M, Li A, et al. DAMGO in the central amygdala alleviates the affective dimension of pain in a rat model of inflammatory hyperalgesia. Neuroscience. 2013;252:359-366.

18. Borges G, Neto F, Mico JA, Berrocoso E. Reversal of monoarthritisinduced affective disorders by diclofenac in rats. Anesthesiology. 2014;12(6):1476-1490.

19. Zhang Y, Meng X, Li A, et al. Electroacupuncture alleviates affective pain in an inflammatory pain rat model. Eur J Pain. 2012;16(2):170-181.

20. Dixon WJ. Efficient analysis of experimental observations. Ann Rev Pharmacol Toxicol. 1980;20:441-462.

21. Shepherd JK, Grewal SS, Fletcher A, Bill DJ, Dourish CT. Behavioural and pharmacological characterisation of the elevated "zeromaze" as an animal model of anxiety. Psychopharmacology (Berl). 1994;116(1):56-64. 
22. Hall CS. Emotional behavior in the rat. I. Defecation and urination as measures of individual differences in emotionality. J Comp Psychol. 1934;18(3):385.

23. Gould TD, Dao DT, Kovacsics CE, editors. The open field test. In: Mood and Anxiety Related Phenotypes in Mice. New York: Springer; 2009:1-20.

24. Breivik H, Collett B, Ventafridda V, Cohen R, Gallacher D. Survey of chronic pain in Europe: prevalence, impact on daily life, and treatment. Eur J Pain. 2006;10(4):287-333.

25. Cox DR, Ashby S, Mace JC, et al. The pain-depression dyad and the association with sleep dysfunction in chronic rhinosinusitis. Int Forum Allergy Rhinol. 2017;7(1):56-63.

26. Zussy C, Gomez-Santacana X, Rovira X, et al. Dynamic modulation of inflammatory pain-related affective and sensory symptoms by optical control of amygdala metabotropic glutamate receptor 4. Mol Psychiatry. 2017;31:59.
27. Sawada A, Niiyama Y, Ataka K, Nagaishi K, Yamakage M, Fujimiya $\mathrm{M}$. The suppression of bone marrow-derived microglia in the amygdala improves the anxiety-like behavior induced by chronic partial sciatic nerve ligation in mice. Pain. 2014;155(9):1762-1772.

28. Shalini SM, Herr DR, Ong WY. The analgesic and anxiolytic effect of souvenaid, a novel nutraceutical, is mediated by alox 15 activity in the prefrontal cortex. Mol Neurobiol. 2016.

29. Sestakova N, Puzserova A, Kluknavsky M, Bernatova I. Determination of motor activity and anxiety-related behaviour in rodents: methodological aspects and role of nitric oxide. Interdiscip Toxicol. 2013;6(3):126-135.

30. Faraji J, Soltanpour N, Jafari SY, et al. Stress inhibits psychomotor performance differently in simple and complex open field environments. Horm Behav. 2014;65(1):66-75.

\section{Journal of Pain Research}

\section{Publish your work in this journal}

The Journal of Pain Research is an international, peer reviewed, open access, online journal that welcomes laboratory and clinical findings in the fields of pain research and the prevention and management of pain. Original research, reviews, symposium reports, hypothesis formation and commentaries are all considered for publication.

\section{Dovepress}

The manuscript management system is completely online and includes a very quick and fair peer-review system, which is all easy to use. Visit http://www.dovepress.com/testimonials.php to read real quotes from published authors. 